\title{
Magnetic field effects on spin relaxation in heterostructures
}

\author{
M.M. Glazov* \\ A. F. Ioffe Physico-Technical Institute, Russian Academy of Sciences, 194021 St. Petersburg, Russia
}

\begin{abstract}
Effect of magnetic field on electron spin relaxation in quantum wells is studied theoretically. We have shown that Larmor effect and cyclotron motion of carriers can either jointly suppress D'yakonov-Perel' spin relaxation or compensate each other. The spin relaxation rates tensor is derived for any given direction of the external field and arbitrary ratio of bulk and structural contributions to spin splitting. Our results are applied to the experiments on electron spin resonance in SiGe heterostructures, and enable us to extract spin splitting value for such quantum wells.
\end{abstract}

\section{INTRODUCTION}

Spin dynamics of charge carriers in semiconductors and semiconductor nanostructures attract much attention during the last decade. Theoretical and experimental efforts are made in field of creation, conservation and manipulation of spin polarized electrons. In this regard the control of spin dephasing processes is a task of prime importance.

The main spin relaxation mechanism in high quality semiconductor quantum wells is that proposed by D'yakonov and Perel'1,2: electrons lose their initial spin during free motion between scattering events due to wavevector dependent effective magnetic field 3.4 This field is determined by the conduction band spin splitting in the systems lacking an inversion center. In bulk zinc-blende lattice semiconductors the spin splitting is cubic in electron wavevector $\boldsymbol{k}$ (Dresselhaus term), and in quantum wells it can be presented as a sum of linear

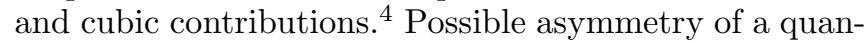
tum well gives rise to an additional linear in wavevector contribution to the spin splitting (Rashba term) 5 These two bulk and structural asymmetry terms have different symmetrical properties and lead to the giant spin relaxation anisotropy in the heterostructure plane ${ }^{2}$. The similar situation is observed in SiGe quantum wells: although bulk Si and Ge possess inversion center, quantum wells grown from these materials can lack such a center and allow the spin splitting of the electronic subbands which can have symmetry of Dresselhaus or Rashba terms. 6

One of the main features of D'yakonov-Perel' spin relaxation mechanism is that it can be suppressed by all processes which change electron wavevector such as scattering from impurities, interface microroughness, phonons, electron-electron collisions 7 and because of cyclotron motion of carriers in the external magnetic field 8 Besides, magnetic field slows down spin relaxation due to Larmor spin precession around the external field direction. ${ }^{8.9}$ In Ref. 10 it was shown that depending on type of linear in $\boldsymbol{k}$ terms, the interference between the Larmor and cyclotron effects will result in either suppression of spin relaxation due to joint contributions of both effects or to their mutual compensation leading to partial or full recovery of spin relaxation rate. However, expressions presented in Ref. 10 were obtained in the limit when only one contribution to the spin splitting is present, moreover as we will see below they predict incorrect dependence of the transverse relaxation time $T_{2}$ on magnetic field orientation.

The aim of present paper is to develop a consistent theory of D'yakonov-Perel' spin relaxation in classical magnetic fields in presence of both Dresselhaus and Rashba splittings. In contrast to Ref. 10 the expressions for all components of spin relaxation tensor will be obtained. When both Dresselhaus and Rashba terms are comparable in magnitude the spin relaxation in the quantum well plane is strongly anisotropic and correct definitions of longitudinal $T_{1}$ and transverse $T_{2}$ spin relaxation times is required. We present an expression for spin susceptibility with allowance for the relaxation anisotropy and show how electron spin resonance (ESR) linewidth is connected with spin relaxation tensor components. We analyse recent experiments on ESR in SiGe quantum wells 10 and extract electron spin splitting from the angular dependence of the linewidth.

\section{THEORY}

Spin-dependent contribution $\mathcal{H}_{S O}$ in the effective Hamiltonian consists of two components. First, in the asymmetrical quantum wells there exists so-called Rashba term 5

$$
\mathcal{H}_{R}=\alpha\left(\sigma_{x} k_{y}-\sigma_{y} k_{x}\right),
$$

where constant $\alpha$ is nonzero due to the heteropotential asymmetry, $\sigma_{i}(i=x, y, z)$ are Pauli matrices. Axes $x$ and $y$ are directed along [100] and [010] respectively.

Second contribution has a Dresselhaus form 4

$$
\mathcal{H}_{D}=\beta_{1}\left(\sigma_{y} k_{y}-\sigma_{x} k_{x}\right)+\beta_{3}\left(\sigma_{x} k_{x} k_{y}^{2}-\sigma_{y} k_{y} k_{x}^{2}\right) .
$$

In the quantum wells grown from zinc-blende lattice materials $\beta_{3}$ is the bulk spin-orbit constant 1.2.4 Even if $\beta_{3}=0$ (like in SiGe quantum wells) $\beta_{1}$ is nonzero due to the asymmetry of chemical bonds at heterointerfaces ${ }^{6.11}$

Sum of Rashba (11) and Dresselhaus-like (2) terms can be conveniently represented as

$$
\mathcal{H}_{S O}=\mathcal{H}_{D}+\mathcal{H}_{R}=\frac{\hbar}{2} \boldsymbol{\sigma} \cdot \boldsymbol{\Omega}_{\boldsymbol{k}},
$$

where components of vector $\boldsymbol{\sigma}$ are Pauli matrices and $\boldsymbol{\Omega}_{\boldsymbol{k}}$ is the effective Larmor frequency of spin precession 
in the spin-splitting induced field. One can see that vector $\boldsymbol{\Omega}_{\boldsymbol{k}}$ contains both first and third harmonics of the angle between the wavevector $\boldsymbol{k}$ and $x$-axis. Spin precession around $\boldsymbol{\Omega}_{\boldsymbol{k}}$ results in spin relaxation while processes changing electron wavevector such as scattering and cyclotron motion slow it down.

The application of the external magnetic field $\boldsymbol{B}$ leads to (a) cyclotron motion of carriers in the quantum well plane, its frequency is given by

$$
\omega_{\mathrm{C}}=\frac{e B_{z}}{m c}
$$

where $B_{z}$ is normal component of magnetic field, $e$ and $c$ are elementary charge and light velocity, $m$ is the electron effective mass and (b) Larmor precession of electron spins with angular velocity $\boldsymbol{\omega}_{\mathrm{L}}$. The components of $\boldsymbol{\omega}_{\mathrm{L}}$ can be presented as

$$
\omega_{\mathrm{L}, i}=\frac{\mu_{B}}{\hbar} g_{i j} B_{j},
$$

where $\mu_{B}=e \hbar / 2 m_{0} c$ is Bohr magneton, $m_{0}$ is free electron mass and $g_{i j}$ are the components of the $g$-factor tensor for electrons in the quantum well, and summation over the repeated indices is omitted. The direction of the vector $\boldsymbol{\omega}_{\mathrm{L}}$ defines the direction of Larmor precession axis. In what follows it is convenient to introduce vector $\boldsymbol{\omega}_{\mathrm{C}}$ directed along the growth axis with absolute value being equal to the cyclotron frequency.

We will use density matrix formalism in order to describe spin relaxation. Electron spin density matrix can be decomposed as

$$
\rho_{k}=f_{k}+s_{k} \cdot \sigma
$$

where $f_{\boldsymbol{k}}$ is the spin-averaged occupation of the $\boldsymbol{k}$ state and $s_{\boldsymbol{k}}$ is the spin vector for electrons in this state. Kinetic equation for spin distribution $\boldsymbol{s}_{\boldsymbol{k}}$ can be written in the following form 7.8

$$
\frac{\partial s_{k}}{\partial t}+s_{k} \times\left(\omega_{L}+\Omega_{k}\right)+\hat{\Lambda} s_{k}+Q\left\{s_{k}\right\}=0,
$$

where the second term describes spin precession in the presence of both external field and spin splitting induced effective magnetic field Eq. (3), the third term comes from wavevector variations under cyclotron motion with operator $\hat{\boldsymbol{\Lambda}}$ defined according to $\left(\hat{\boldsymbol{\Lambda}} \boldsymbol{s}_{\boldsymbol{k}}\right)_{i}=$ $\boldsymbol{\omega}_{\mathrm{C}}\left[\boldsymbol{k} \times \partial s_{\boldsymbol{k}, i} / \partial \boldsymbol{k}\right]$, and the last term is the collision integral. For simplicity we will consider only elastic scattering processes and describe them using two times $\tau_{1}$ and $\tau_{3}$, responsible for relaxation of first and third harmonics of distribution function ${ }^{2}$ Eq. (6) is valid for classical magnetic fields, quantum effects on spin relaxation were discussed in Refs. 1213 for strong and weak fields respectively.

Considering spin-splitting as a small perturbation $\left(\Omega_{\boldsymbol{k}} \tau_{1}, \Omega_{\boldsymbol{k}} \tau_{3} \ll 1\right)$ one can present spin distribution function $\boldsymbol{s}_{\boldsymbol{k}}$ as

$$
s_{k}=s_{k}^{0}+\delta s_{k}
$$

where $s_{\boldsymbol{k}}^{0}$ is quasi-equilibrium distribution function and $\delta s_{\boldsymbol{k}}$ is a non-equilibrium correction arising due to spin splitting. After summation (6) over the wavevectors we arrive to the balance equation describing relaxation of the total spin $S=\sum_{k} s_{k}$

$$
\frac{d \boldsymbol{S}}{d t}+\boldsymbol{S} \times \boldsymbol{\omega}_{\mathrm{L}}+\hat{\boldsymbol{\Gamma}} \boldsymbol{S}=0
$$

where the tensor of inverse spin relaxation times $\hat{\boldsymbol{\Gamma}}$ is defined according to

$$
\hat{\Gamma} S=\sum_{k} \delta s_{k} \times \Omega_{k}
$$

and the non-equilibrium correction $\delta \boldsymbol{s}_{\boldsymbol{k}}$ should be determined from the solution of Eq. (6).

We will be interested in the case of strong enough magnetic field : $\Omega_{\boldsymbol{k}}^{2} \tau_{1}, \Omega_{\boldsymbol{k}}^{2} \tau_{3} \ll \omega_{\mathrm{L}}$. Then all non-diagonal components of the tensor $\hat{\boldsymbol{\Gamma}}$ associating spin relaxation along external field and in the transverse plane are zero. Noting that the first and third harmonics of spin distribution have independent dynamics we present $\hat{\Gamma}$ in the form

$$
\hat{\boldsymbol{\Gamma}}=\hat{\boldsymbol{\Gamma}}^{(1)}+\hat{\boldsymbol{\Gamma}}^{(3)}
$$

where the upper index denotes the number of angular harmonic.

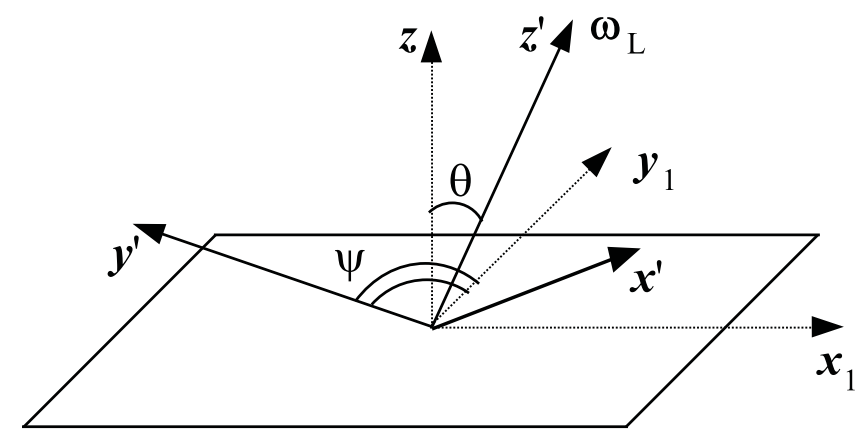

FIG. 1: Frames of axes. $z \|[001]$ is a growth axis. Axes $x_{1} \|[1 \overline{1} 0]$ and $y_{1} \|[110]$. The frame of axis $x^{\prime}, y^{\prime}$ and $z^{\prime}$ is connected with the external magnetic field: $z^{\prime} \| \boldsymbol{\omega}_{\mathrm{L}}, y^{\prime}$ is a perpendicular to $\boldsymbol{\omega}_{\mathrm{L}}$ lying in a quantum well plane, and $x^{\prime}$ is chosen so that $x^{\prime}, y^{\prime}$ and $z^{\prime}$ form a right-hand triple.

It is convenient to present components of spin relaxation tensor in the new frame of coordinates with $z^{\prime}$ axis directed along Larmor frequency vector $\boldsymbol{\omega}_{\mathrm{L}}$, and $y^{\prime}$ axis lying in the quantum well plane and being perpendicular to the $\boldsymbol{\omega}_{\mathrm{L}}$ (Fig. 1). Let $\theta$ be the angle between $z^{\prime}$ and $z$ and $\psi$ be the angle between $y^{\prime}$ and $y_{1} \|[110]$. One can show that for electrons with fixed energy $E$ the non-zero components of tensors $\hat{\boldsymbol{\Gamma}}^{(1)} \hat{\boldsymbol{\Gamma}}^{(3)}$ can be written as 


$$
\begin{gathered}
\Gamma_{z^{\prime} z^{\prime}}^{(1)}=\frac{2 \tau_{1} k^{2}}{\hbar^{2}} \frac{N_{1, z^{\prime} z^{\prime}}}{D_{1,+} D_{1,-}}, \quad \Gamma_{i i}^{(1)}=\frac{2 \tau_{1} k^{2}}{\hbar^{2}} \frac{N_{1, i i}}{1+\omega_{\mathrm{C}}^{2} \tau_{1}^{2}}, \quad \Gamma_{x^{\prime} y^{\prime}}^{(1)}=-\frac{4 \tau_{1} k^{2} \alpha \beta}{\hbar^{2}} \frac{\cos \theta \sin 2 \psi}{1+\omega_{\mathrm{C}}^{2} \tau_{1}^{2}} \\
\Gamma_{z^{\prime} z^{\prime}}^{(3)}=\frac{2 \tau_{3} k^{6} \beta_{3}^{2}}{8 \hbar^{2}} \frac{N_{3, z^{\prime} z^{\prime}}}{D_{3,-}}, \quad \Gamma_{x^{\prime} x^{\prime}}^{(3)}=\frac{\tau_{3} k^{6} \beta_{3}^{2}}{8 \hbar^{2}}\left\{\frac{1}{1+9 \omega_{\mathrm{C}}^{2} \tau_{3}^{2}}+\frac{\sin ^{2} \theta\left[1+\left(\omega_{\mathrm{L}}^{2}+9 \omega_{\mathrm{C}}^{2}\right) \tau_{3}^{2}\right]}{D_{3,+} D_{3,-}}\right\} \\
\Gamma_{y^{\prime} y^{\prime}}^{(3)}=\frac{\tau_{3} k^{6} \beta_{3}^{2}}{8 \hbar^{2}\left(1+9 \omega_{\mathrm{C}}^{2} \tau_{3}^{2}\right)}\left\{1-\frac{\sin ^{2} \theta\left[1+\left(\omega_{\mathrm{L}}^{2}-27 \omega_{\mathrm{C}}^{2}\right) \tau_{3}^{2}\right] \omega_{\mathrm{L}}^{2} \tau_{3}^{2}}{D_{3,+} D_{3,-}}\right\}
\end{gathered}
$$

Here $k=\sqrt{2 m E} / \hbar, i=x^{\prime}, y^{\prime}, \beta=\beta_{1}-\beta_{3} k^{2} / 4$ is a coefficient at first harmonics in Dresselhaus splitting (2), $D_{j, \pm}=1+\left(\omega_{\mathrm{L}} \pm j \omega_{\mathrm{C}}\right)^{2} \tau_{j}^{2}$, where $j=1,3$, and

$$
\begin{gathered}
N_{1, z^{\prime} z^{\prime}}=\alpha^{2}\left[1+\cos ^{2} \theta+\left(\omega_{\mathrm{C}}+\omega_{\mathrm{L}} \cos \theta\right)^{2} \tau_{1}^{2}+\left(\omega_{\mathrm{C}} \cos \theta+\omega_{\mathrm{L}}\right)^{2} \tau_{1}^{2}\right] \\
+\beta^{2}\left[1+\cos ^{2} \theta+\left(\omega_{\mathrm{C}}-\omega_{\mathrm{L}} \cos \theta\right)^{2} \tau_{1}^{2}+\left(\omega_{\mathrm{C}} \cos \theta-\omega_{\mathrm{L}}\right)^{2} \tau_{1}^{2}\right]+2 \alpha \beta \cos 2 \psi \sin ^{2} \theta\left[1+\left(\omega_{\mathrm{C}}^{2}+\omega_{\mathrm{L}}^{2}\right) \tau_{1}^{2}\right], \\
\left.N_{1, x^{\prime} x^{\prime}}=\left(\alpha^{2}+\beta^{2}\right)\left\{1+\sin ^{2} \theta\left(1+\omega_{\mathrm{C}}^{2} \tau_{1}^{2}\right)\left[1+\left(\omega_{\mathrm{C}}^{2}+\omega_{\mathrm{L}}^{2}\right) \tau_{1}^{2}\right)\right]\left(D_{1,+} D_{1,-}\right)^{-1}\right\}+2 \alpha \beta \cos 2 \psi, \\
N_{1, y^{\prime} y^{\prime}}=\left(\alpha^{2}+\beta^{2}\right)\left\{1-\sin ^{2} \theta\left[1+\left(\omega_{\mathrm{L}}^{2}-3 \omega_{\mathrm{C}}^{2}\right) \tau_{1}^{2}\right]\left(D_{1,+} D_{1,-}\right)^{-1} \omega_{\mathrm{L}}^{2} \tau_{1}^{2}\right\}-2 \alpha \beta \cos 2 \psi, \\
N_{3, z^{\prime} z^{\prime}}=1-\sin ^{2} \frac{\theta}{2}\left\{1+\left[\cos \theta\left(1+\left(\omega_{\mathrm{L}}^{2}+9 \omega_{\mathrm{C}}^{2}\right) \tau_{3}^{2}\right)-6 \omega_{\mathrm{L}} \omega_{\mathrm{C}} \tau_{3}^{2}\right] D_{3,+}^{-1}\right\} .
\end{gathered}
$$

Eqs. (10) and (11) describe spin relaxation of two-dimensional electron gas subject to classical magnetic field. These formulae are valid for arbitrary ratio of Dresselhaus and Rashba contributions and for arbitrary orientation of the external field. Generalization of Eqs. (10), (11) for the case of any given energy distribution of electrons can be carried out in the standard way ${ }^{2}$

\section{RESULTS AND DISCUSSION}

\section{A. Longitudinal relaxation}

We will start the analysis of the expressions Eqs. (10) and (11) for the spin relaxation tensor components in the case when magnetic field is normal to the quantum well structure, i.e. $\theta=0$. Then the expression for the longitudinal spin relaxation rate $1 / T_{1}=\Gamma_{z z}=\Gamma_{z z}^{(1)}+\Gamma_{z z}^{(3)}$ reduces to

$$
\frac{1}{T_{1}}=\frac{4 \tau_{1} k^{2}}{\hbar^{2}}\left[\frac{\alpha^{2}}{1+\left(\omega_{\mathrm{L}}-\omega_{\mathrm{C}}\right)^{2} \tau_{1}^{2}}+\frac{\beta^{2}}{1+\left(\omega_{\mathrm{L}}+\omega_{\mathrm{C}}\right)^{2} \tau_{1}^{2}}\right]+\frac{\tau_{3} k^{6} \beta_{3}^{2}}{4 \hbar^{2}} \frac{1}{1+\left(\omega_{\mathrm{L}}-3 \omega_{\mathrm{C}}\right)^{2} \tau_{3}^{2}} .
$$

Eq. (12) shows that external magnetic field has different effects on spin relaxation depending on the dominant type of spin splitting. When Rashba term is dominant $\left(\beta_{3}=0, \beta=0, \alpha \neq 0\right)$ and electron $g$-factor is positive the Larmor and cyclotron effects partially compensate each other. In the case when first harmonics in Dresselhaus splitting is dominant $\left(\beta \gg \beta_{3} k^{2}, \alpha=0\right)$ both Larmor and cyclotron effects jointly slow down spin re- laxation. If the spin splitting is dominated by the third harmonics then Larmor and cyclotron effects compensate each other.

Such interference behavior is a result of the fact that according to Eq. (8) spin relaxation rate is determined by the vector product of nonequilibrium correction $\delta \boldsymbol{s}_{\boldsymbol{k}}$ and effective spin precession frequency $\boldsymbol{\Omega}_{\boldsymbol{k}}$. If no magnetic field is applied then $\delta \boldsymbol{s}_{\boldsymbol{k}}$ is orthogonal to $\boldsymbol{\Omega}_{\boldsymbol{k}}$ and 


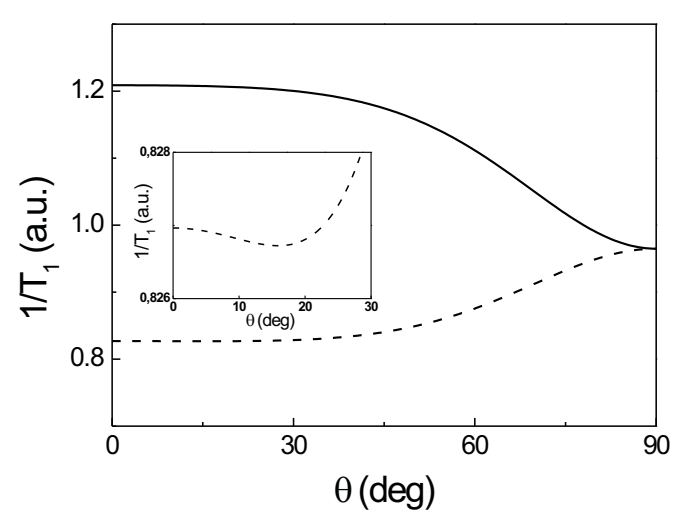

FIG. 2: Longitudinal spin relaxation rate vs. magnetic field tilt angle $\theta$ for SiGe based quantum well. Spin relaxation rate is measured in the units $2 \tau_{1} k^{2} \delta^{2} / \hbar^{2}$ where $\delta$ is a coefficient at the first harmonics of spin splitting. Solid curve corresponds to Rashba term $(\alpha=\delta, \beta=0)$, dashed curve to Rashba term $(\alpha=0, \beta=\delta)$. The contribution of the third harmonics is neglected. At $\theta=0$ the parameter $\omega_{\mathrm{C}} \tau_{1}=1$. The parameters of calculation were taken from Ref. 1: $g=2, m=0.196 m_{0}$. Inset shows nonmonotonous behavior of $1 / T_{1}(\theta)$ when Dresslhaus term dominates.

spin relaxation rate is fastest. External magnetic field results in the precession of in-plane spin component $\delta \boldsymbol{s}_{\boldsymbol{k}}$ around Larmor vector $\boldsymbol{\omega}_{\mathrm{L}}$ and in cyclotron rotation of the $\boldsymbol{\Omega}_{\boldsymbol{k}}$. If these rotations are not synchronous then vectors $\delta \boldsymbol{s}_{\boldsymbol{k}}$ and $\boldsymbol{\Omega}_{\boldsymbol{k}}$ are no longer orthogonal and spin relaxation rate becomes slower. In the case of synchronous rotation of in-plane spin and effective magnetic field the spin relaxation is not suppressed. It is important to note that the direction of cyclotron rotation of $\boldsymbol{\Omega}_{\boldsymbol{k}}$ depends on the dominant spin splitting mechanism. If Rashba term is largest then effective magnetic field $\boldsymbol{\Omega}_{\boldsymbol{k}}$ is perpendicular to the wavevector and rotates in the same direction as $\boldsymbol{k}$. Thus, spin relaxation becomes slower by the factor of $1+\left(\omega_{\mathrm{L}}-\omega_{\mathrm{C}}\right)^{2} \tau_{1}^{2}$ and Larmor and cyclotron effects partially compensate each other provided electron $g$-factor being positive. If spin splitting is due to the first harmonics of Dresselhaus term then cyclotron rotation of $\boldsymbol{\Omega}_{\boldsymbol{k}}$ and Larmor precession at positive $g$-factor have opposite directions and slow down spin relaxation by the factor of $1+\left(\omega_{\mathrm{L}}+\omega_{\mathrm{C}}\right)^{2} \tau_{1}^{2}$. When third harmonics dominates, the rotation of $\boldsymbol{\Omega}_{\boldsymbol{k}}$ and Larmor precession take place in the same direction but the rotation frequency of $\boldsymbol{\Omega}_{\boldsymbol{k}}$ is three times higher than cyclotron one.

Figure 2 shows longitudinal spin relaxation rate $1 / T_{1}=$ $\Gamma_{z^{\prime} z^{\prime}}$ dependence on the magnetic field tilt angle $\theta$ calculated for SiGe structure. The case of dominant Rashba term is shown by a solid line, and dashed line presents the results when spin splitting is determined by Dresselhaus term. The contribution of the third harmonics is neglected in all calculations. The qualitatively different angular dependencies of $1 / T_{1}$ are clearly seen. When

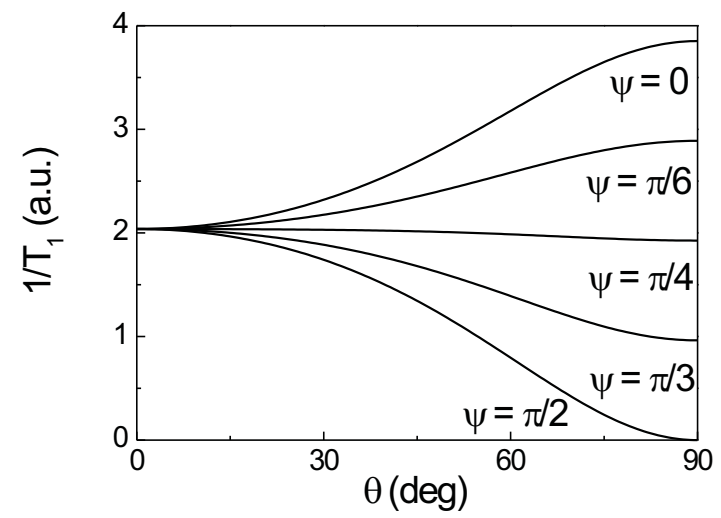

FIG. 3: Longitudinal spin relaxation rate vs. magnetic field tilt angle $\theta$ in the case of equal Dresselhaus and Rashba terms $(\alpha=\beta)$ for different orientations of magnetic field. Spin relaxation rate is presented in units $2 \tau_{1} k^{2} \alpha^{2} / \hbar^{2}$. The values of parameters used in the calculation are the same as for Fig. 2

Rashba contribution is a main one the spin relaxation rate decreases with increase of in-plane component of magnetic field because tilting of the field results in the decrease of the cyclotron frequency $\omega_{\mathrm{C}}$. When Dresselhaus term is dominant the dependence $1 / T_{1}(\theta)$ is nonmonotonous (see inset): first, spin relaxation slows down owing to the decrease of projection of $\boldsymbol{\Omega}_{\boldsymbol{k}}$ on the plane perpendicular to the magnetic field. At larger $\theta$ spin relaxation accelerates due to cyclotron frequency decrease.

Figure 3 shows longitudinal spin relaxation rate for different orientations of magnetic field in the case when Rashba and Dresselhaus terms have equal strengths $(\alpha=$ $\beta)$. Different curves correspond to different angles between magnetic field and main axes of the structure. One can see that spin relaxation rate is highest at $\psi=0$, i.e. for magnetic field inclined to the $x_{1} \|[1 \overline{1} 0]$ axis and is lowest at $\psi=\pi / 2$ (magnetic field inclined to $y_{1} \|[110]$ ). This result is a direct consequence of the interference of linear in $\boldsymbol{k}$ spin dependent terms resulting in the suppression at $\alpha=\beta$ of the spin splitting along $[1 \overline{1} 0]^{2} \stackrel{2}{*}$ For the same reason spin relaxation rate calculated with allowance for the first harmonics of the wavevector only goes to zero when magnetic field is directed along [110], the account for the third harmonics in spin splitting leads to the finite spin lifetime along $y_{1} \stackrel{2}{*}$

Different behavior of spin relaxation rate for different values of $\psi$ can be explained taking into account that in normal magnetic field spin relaxation is suppressed mostly by cyclotron effect since $\omega_{\mathrm{L}} / \omega_{\mathrm{C}}=g m / 2 m_{0} \approx 0.2$. With increase of $\theta$ cyclotron frequency decreases, and if $-\pi / 4 \leq \psi \leq \pi / 4$ effective spin splitting increases. Therefore spin relaxation becomes faster if $-\pi / 4 \leq \psi<\pi / 4$. At $\psi=\pi / 4$ and $\omega_{\mathrm{C}} \tau_{1}=1$ spin relaxation rate should not depend of tilt angle if one completely neglects Larmor effect. With tilting of magnetic field cyclotron frequency 
(a)

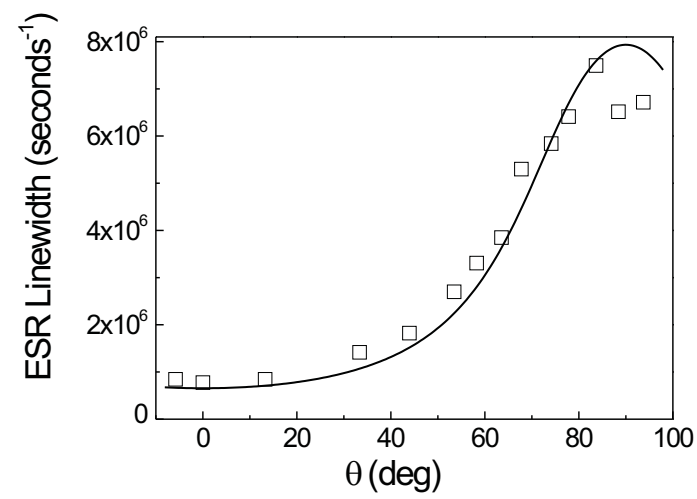

(b)

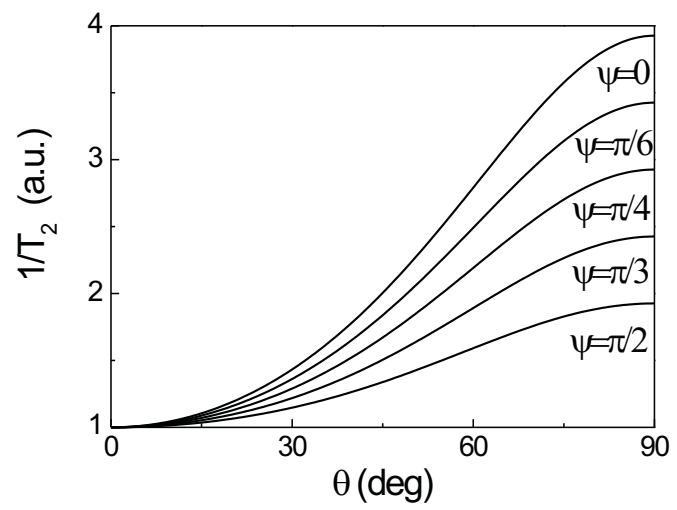

FIG. 4: Transverse relaxation time vs. magnetic field tilt angle $\theta$ for SiGe structure. (a) Open squares show ESR linewidth measured in Ref. 10. Solid curve presents the results of calculation when Rashba term dominates. Parameters $\omega_{\mathrm{C}}=2.96 \times$ $10^{11} \mathrm{~s}^{-1}$ at normal magnetic field orientation, $\omega_{\mathrm{L}}=5.93 \times 10^{10} \mathrm{~s}^{-1}$, and momentum relaxation time $\tau_{1}=10^{-11} \mathrm{~s}$ were taken from experiment. Spin precession frequency is $\Omega_{k_{F}}=\alpha k_{F} / \hbar=4 \times 10^{8} \mathrm{~s}^{-1}$. (b) Dresselhaus and Rashba terms are equal $(\alpha=\beta)$. Spin relaxation rate is measured in the units of $\tau_{1} k^{2} \alpha^{2} / \hbar^{2}$. Parameters of calculation are the same as for Fig. 2a.

decreases and Larmor effect becomes more important resulting in slight decrease of relevant curve $(\psi=\pi / 4)$. If $\pi / 4<\psi \leq 3 \pi / 4$ then competition between decrease of $\omega_{\mathrm{C}}$, decrease of effective spin splitting and Larmor effect is possible leading to nonmonotonous angular dependence of the spin relaxation rate. However at $\omega_{\mathrm{C}} \tau_{1}=1$ taken for calculation presented in Fig Bb spin relaxation slows down.

\section{B. Transverse relaxation}

Now we turn to the analysis of spin relaxation in the plane perpendicular to the external field. In the ESR experiments the spin susceptibility is measured which in the limit of low microwave power has a following form

$$
\chi \propto \frac{1}{\omega_{\mathrm{L}}-\omega+\mathrm{i} / T_{2}}
$$

where $\omega$ is microwave frequency and $T_{2}$ is a transverse relaxation time. Eq. (13) is valid when microwave frequency is close to Larmor one, small renormalization of resonance frequency $\sim T_{2}^{-1}$ is neglected. Transverse relaxation time can be expressed through the components of spin relaxation tensor as

$$
1 / T_{2}=\frac{\Gamma_{x^{\prime} x^{\prime}}+\Gamma_{y^{\prime} y^{\prime}}}{2},
$$

and ESR half-maximum full width $\Delta \omega=2 / T_{2}$. Eq. (14) generalizes results of Refs. 1014 for the case of arbitrary orientation of magnetic field and anisotropic spin relaxation. One can see that the combination $\Gamma_{x^{\prime} x^{\prime}}+\Gamma_{y^{\prime} y^{\prime}}$ is invariant to the choice of axes in the transverse plane, therefore resonant peak width is independent of microwave field polarization.
If the external field is oriented along the quantum well growth axis then $1 / T_{2}$ has the form

$$
1 / T_{2}=\frac{2 \tau_{1} k^{2}}{\hbar^{2}} \frac{\alpha^{2}+\beta^{2}}{1+\omega_{\mathrm{C}}^{2} \tau_{1}^{2}}+\frac{\tau_{3} k^{6}}{8 \hbar^{2}} \frac{\gamma^{2}}{1+9 \omega_{\mathrm{C}}^{2} \tau_{1}^{2}} .
$$

In the particular case of $\beta=0$ and $\omega_{\mathrm{C}}=0$ this expression coincides with equations obtained in Ref. 14. However it is in sharp disagreement with results of Ref. 10, where zero spin relaxation rate is predicted for normal magnetic field. Those results were based on the assumption that transverse spin relaxation is governed by the normal component of $\boldsymbol{\Omega}_{\boldsymbol{k}}$. This assumption is invalid, since spin relaxation along, say, $x^{\prime}$ is governed by both $\Omega_{\boldsymbol{k}, y^{\prime}}$ and $\Omega_{\boldsymbol{k}, z^{\prime}}$.

Figure 4 shows the results of calculation of the transverse spin relaxation rate $1 / T_{2}$ in SiGe structure versus external magnetic field orientation. The transverse relaxation rate monotonously increase with tilt angle (solid curve on Fig. 四). It comes from the fact that spin relaxation in the plane normal to magnetic field is suppressed owing to cyclotron effect, see Eqs. (10) and (15). Cyclotron frequency is maximal if magnetic field is normal to the quantum well plane and decreases proportionally to $\cos \theta$ with tilting magnetic field.

Open squares on Fig. 4a show the ESR linewidth measured in SiGe quantum well structure ${ }^{10}$ The parameters of calculation were taken equal to the experimental ones: $\omega_{\mathrm{C}}=2.96 \times 10^{11} \mathrm{~s}^{-1}$ at normal magnetic field, $\omega_{\mathrm{L}}=5.93 \times 10^{10} \mathrm{~s}^{-1}$, the momentum relaxation time $\tau_{1}=10^{-11} \mathrm{~s}$. We used the effective frequency of spin precession on the Fermi level $\Omega_{k_{F}}=\alpha k_{F} / \hbar$ as a fitting parameter. The best agreement is at $\Omega_{k_{F}}=4 \times 10^{8} \mathrm{~s}^{-1}$. The order of magnitude of $\Omega_{k_{F}}$ agrees with estimations of Ref. 14. Obtained splitting is lower than value $6.3 \times 10^{8} \mathrm{~s}^{-1}$ presented in Ref. 10, because fitting in 
that paper was done with incorrect dependence $1 / T_{2}(\theta)$.

In the case when Dresselhaus and Rashba terms are equal in magnitude $(\alpha=\beta)$, the dependence of transverse relaxation rate $1 / T_{2}(\theta)$ on magnetic field tilt angle is qualitatively the same. The slope depends on the inplane rotation angle $\psi$ reflecting anisotropy of the system.

In conclusion, we have studied in detail effects of magnetic field on electron spin relaxation in SiGe quantum wells. We have shown that, depending on electron $g$ factor sign and dominant spin splitting mechanism, Larmor and cyclotron effects are either compete with each other or jointly suppress spin relaxation. The expression for ESR linewidth was derived for the case of anisotropic transverse relaxation. Fitting by the derived expressions the experimental data we obtained spin splitting value for SiGe structure studied in Ref. 10.

\section{Acknowledgments}

Author is grateful to L.E. Golub and E.L. Ivchenko for valuable discussions. Work was partially supported by RFBR and scholarship of "Dynasty" foundation ICFPM.
* Electronic address: glazov@coherent.ioffe.ru

1 E.L. Ivchenko and G.E. Pikus, Superlattices and Other Heterostructures: Symmetry and Optical Phenomena, Springer Series in Solid State Sciences, vol. 110, SpringerVerlag, 1995; 2nd edition 1997.

2 N.S. Averkiev, L.E. Golub and M. Willander, J. Phys.: Condens. Matter 14, R271, (2002).

3 M.I. Dyakonov and V.I. Perel, Fiz. Tverd. Tela 13, 3581 (1971) [Sov. Phys.Solid State, 13, 3023 (1971)].

4 M.I. Dyakonov and V.Yu. Kachorovskii, Fiz. Tekh. Poluprov. 20, 178 (1986) [Sov. Phys.Semicond., 20, 110 (1986)].

5 Yu.L. Bychkov and E.I. Rashba, Pis. Zh. Eksp. Teor. Fiz. 39, 66 (1984) [JETP Lett. 39, 78 (1984)].

${ }^{6}$ L.E. Golub and E.L. Ivchenko, Phys. Rev. B 69, 115333 (2004).
7 M.M. Glazov and E.L. Ivchenko, Journal of Supercond. Inc. Novel Magnetism 16, 735.

8 E. L. Ivchenko, Fiz. Tverd. Tela 15, 1566 (1973) [Sov. Phys. Solid State 15, 1048 (1973)].

9 F. X. Bronold, I. Martin, A. Saxena and D. L. Smith, Phys. Rev. B 66, 233206 (2002).

10 Z. Wilamowski and W. Jantsch, Phys. Rev. B 69, 035328 (2004).

11 U. Roessler and J. Kainz, Solid State Communs. 121, 313 (2002).

12 A.A. Burkov and L. Balents, cond-mat/0401398 (2004).

13 I.S. Lyubinsky and V.Yu. Kachorovskii, cond-mat/0404391 (2004).

14 C. Tahan and R. Joynt, cond-mat/0401615 (2004). 